\title{
Implementação de um projeto de avaliação da Rede de Cuidados à Pessoa com Deficiência
}

\section{Implementation of an evaluation project of the Disabled People Care Network}

\author{
La implementación de un proyecto \\ de evaluación de la Red de Atención \\ de Personas con Discapacidad
}

\author{
Andrezza Gonzalez Escarce* \\ Amélia Augusta de Lima Friche* \\ Roberta Alvarenga Reis* \\ Mônica Farina Neves Santos* \\ Gabriela Cintra Januário* \\ Fernanda Jorge Maciel* \\ Raimundo de Oliveira Neto* \\ Stela Maris Aguiar Lemos*
}

\section{Resumo}

O objetivo do estudo é descrever o percurso utilizado por uma equipe de pesquisadores para elaboração e desenvolvimento de um projeto de pesquisa para avaliação da Rede de Cuidados à Pessoa com Deficiência em Minas Gerais. Trata-se de relato de experiência, no qual foram destacadas as etapas percorridas pela equipe e a importância da discussão do tema da pesquisa, bem como a realização de reuniões periódicas;

\footnotetext{
*Universidade Federal de Minas Gerais, Belo Horizonte, MG, Brasil.

Financiamento: Fundação de Amparo à Pesquisa do Estado de Minas Gerais (FAPEMIG) - Edital 14/2012 - Programa de Pesquisa para o SUS - PPSUS-REDE.

Contribuição dos autores:

AGE: concepção e delineamento do estudo, análise e interpretação dos dados, redação e aprovação final da versão a ser publicada. AALM: concepção e delineamento do estudo, análise e interpretação dos dados, redação, revisão e aprovação final da versão a ser publicada. RAR: análise e interpretação dos dados, redação, revisão e aprovação final da versão a ser publicada. MFNS, GCJ: análise e interpretação dos dados, redação e revisão da versão a ser publicada. FJM: análise e interpretação dos dados, redação, revisão e aprovação final da versão a ser publicada. RON: análise e interpretação dos dados, redação e revisão da versão a ser publicada. SMAL: concepção e delineamento do estudo, análise e interpretação dos dados, redação, revisão e aprovação final da versão a ser publicada.
}

E-mail para correspondência: Andrezza Gonzalez Escarce - andrezza.ge@gmail.com Recebido: 11/10/2016

Aprovado: 23/05/2017 
dos resultados preliminares com base no estudo dos dados secundários; da elaboração dos instrumentos de coleta; da seleção de novos integrantes e realização da instrumentalização; da realização do cálculo amostral e do estudo piloto. É de relevância o registro deste percurso para que novos estudos possam reproduzir este processo e, consequentemente, gerar a comparação com resultados obtidos em outros cenários.

Palavras-chave: Metodologia; Avaliação; Sistema Único de Saúde; Atenção à saúde.

\section{Abstract}

This study is aiming to describe the course taken by a team of researchers to elaborate and develop a research project to evaluate the Disabled People Care Network in the state of Minas Gerais. This is an experience report, in which the stages taken by the team were highlighted and the importance upon the discussion of the research topic, as well as the regular meetings occurrences; preliminary results based on the regarding the study of secondary data; the preparation of collection instruments; the selection of new members and holding the instrumentalization; the completion of the sample calculation and the pilot study. It is important to register the route taken in a way to allow new studies to replicate this process and thus generate the comparison with results in different scenarios.

Keywords: Methodology; Evaluation; Unified Health System; Health care.

\section{Resumén}

El objetivo del estudio es describir el recorrido metodológico de un equipo de investigadores a planear y desarrollar un proyecto de investigación para evaluar la Red de cuidado de la persona con discapacidad en Minas Gerais. Se trata de un relato de experiência, dividido por pasos. Fueron aspectos destacados en el manuscrito: las medidas tomadas por el equipo, la discusión del tema de la investigación, las reuniones periódicas; los resultados preliminares sobre la base del estudio de datos secundarios; el desarrollo de los instrumentos de recolecta de datos; la capacitación de nuevos miembros; la realización del cálculo de la muestra y un estudio piloto. Es importante registrar este camino para que nuevos estudios pueden replicar este proceso y por lo tanto generar la comparación con los resultados en otros contextos.

Palabras claves: Metodologia; Evaluación; Sistema Único de Salud; Atención a la salud.

\section{Introdução}

A Organização Mundial de Saúde (OMS), por meio da Classificação Internacional de Funcionalidade, Incapacidade e Saúde (CIF), define deficiência como um problema nas funções ou estruturas do corpo ${ }^{1}$. Dessa forma, a funcionalidade torna-se protagonista, ao invés da doença, e a incapacidade do indivíduo passa a ser vista como um aspecto da sua relação com seu ambiente ${ }^{2-4}$.

De acordo com o Relatório Mundial sobre a Deficiência ${ }^{5}$, do ano de $2012,15,3 \%$ da população mundial apresenta alguma deficiência moderada ou grave. No Brasil, há diferentes estratégias de identificação das pessoas com deficiência. A Pesquisa Nacional de Saúde (PNS) ${ }^{6}$, com base amostral, encontrou que $6,2 \%$ da população apresentava pelo menos uma das deficiências investigadas, sendo a visual a de maior incidência, seguida pela física, auditiva e intelectual. Já o Censo do $\mathrm{IBGE}^{7}$, em 2010, encontrou $23,9 \%$.

Diante dessa realidade, com o objetivo de ampliar e qualificar o atendimento de pessoas com deficiência temporária ou permanente; progressiva, regressiva ou estável; intermitente ou contínua; em abril de 2012, o Ministério da Saúde instituiu a Rede de Cuidados à Pessoa com Deficiência (RCPD) no âmbito do Sistema Único de Saúde (SUS) ${ }^{8}$. Suas ações são direcionadas às pessoas com deficiência auditiva, física, intelectual, ostomia e com múltiplas deficiências ${ }^{8}$.

No entanto, sua construção trata de um processo complexo, muitas vezes entremeado por barreiras tanto socioculturais quanto operacionais. Além disso, o conceito ampliado de saúde associado aos preceitos de integralidade, universalidade e descentralização propostos pelo SUS, tornam imprescindível o conhecimento da estruturação 
dessa Rede nas esferas estaduais, visando, assim, $o$ adequado funcionamento de todo o sistema ${ }^{9}$.

Dessa forma, é importante que a avaliação contemple a Rede em toda sua complexidade, e aborde por diferentes olhares as percepções de gestores, profissionais de saúde e usuários, a fim de contribuir para melhoria da Rede e do serviço prestado. Ressalta-se, ainda, a escassez de metodologias de avaliação e estudos relativos à RCPD ${ }^{10,11}$.

Frente a esse contexto, a presente comunicação caracteriza-se como um relato de experiência elaborado pela equipe do projeto de pesquisa intitulado "Rede de Cuidado à Pessoa com Deficiência em Minas Gerais: perfil populacional, avaliação do acesso e da estrutura, e tem como objetivo descrever o percurso utilizado para elaboração e desenvolvimento de um projeto de pesquisa.

\section{Métodos}

\section{Delineamento do estudo}

$\mathrm{O}$ trabalho delineado constou de um estudo observacional analítico do tipo transversal com o objetivo de descrever o processo de construção das etapas utilizadas para identificar, conhecer e avaliar a RCPD no estado de Minas Gerais na perspectiva dos coordenadores/gestores dos serviços, profissionais e usuários, bem como avaliar a estrutura desses serviços.

O projeto conta com o financiamento da Fundação de Amparo à Pesquisa do Estado de Minas Gerais (FAPEMIG) e foi aprovada pelo Comitê de Ética em Pesquisa da Universidade Federal de Minas Gerais, sob o parecer ETIC CAAE 33703914.8.0000.5149.

\section{Composição da equipe}

A equipe foi formada por três docentes de universidades federais, dos estados de Minas Gerais e do Rio Grande do Sul; dois gestores de saúde da Secretaria de Estado de Minas Gerais (SES/MG); um assessor da direção geral da Escola de Saúde Pública de Minas Gerais (ESP/MG), um profissional da Junta Reguladora de Saúde Auditiva de Belo Horizonte/MG, uma fonoaudióloga bolsista de gestão em ciência e tecnologia do projeto e seis bolsistas que integraram o projeto posteriormente. Cenário do estudo

O cenário do estudo é a RCPD do estado de Minas Gerais, instituída no ano de $2012^{12}$. As ações de reabilitação ocorrem em unidades especializadas e os serviços são articulados entre si, garantindo, assim, a integralidade do acesso em cada ponto de atenção $0^{13}$.

$\mathrm{O}$ estado de Minas Gerais possui $853 \mathrm{mu}-$ nicípios, distribuídos em 77 Regiões de Saúde, divididas em 13 Regiões Ampliadas de Saúde ${ }^{14}$. No ano de 2015, possuía 209 serviços cadastrados, em todas as Regiões Ampliadas de Saúde do Estado em diferentes fases de implementação de uma nova Rede, em processo de adequação do funcionamento e articulação entre os serviços, de acordo com a legislação vigente ${ }^{8,12,13}$.

\section{Delineamento do cálculo amostral e seus resultados}

As unidades de análise definidas foram as Regiões Ampliadas de Saúde e as Regiões de Saúde, de acordo com o Plano Diretor de Regionalização de Minas Gerais, uma vez que esse Plano serve como diretriz organizativa da atenção à saúde no SUS.

Os pontos de atenção que compõem a RCPD foram a base para o cálculo amostral no Estado de Minas Gerais.

A amostragem aleatória foi realizada em três estágios, estratificada pelas 13 regiões ampliadas de saúde e proporcional à população de cada uma delas, conforme demonstrado na figura 1 .

Em cada serviço selecionado, no momento da visita, os usuários foram aleatoriamente convidados a participar. Todos os profissionais e gestores presentes também foram convidados a participar do estudo.

Foram considerados como parâmetros para o cálculo amostral: nível de confiança de $95 \%$, margem de erro de $5 \%$ e a prevalência da variável de interesse (deficiência), segundo dados do Censo Brasileiro de $2010^{7}$, escolhido por se tratar de pesquisa com o universo populacional e ser mais próximo dos valores relatados no Relatório Mundial sobre Deficiência ${ }^{5}$.

Definido o delineamento do cálculo amostral, sua análise foi realizada por meio do método para estimação de proporções para populações finitas ${ }^{15}$, com alocação proporcional da amostra primeiramente pelas 13 regiões ampliadas de saúde e posteriormente pela região de saúde e tipo de serviço.

O tamanho da amostra para estimação de proporções para populações finitas é dado pela expressão abaixo, no qual $\mathbf{Z}_{\alpha} \mathbf{Z}_{\alpha}$ representa o percentil da distribuição normal correspondente ao 


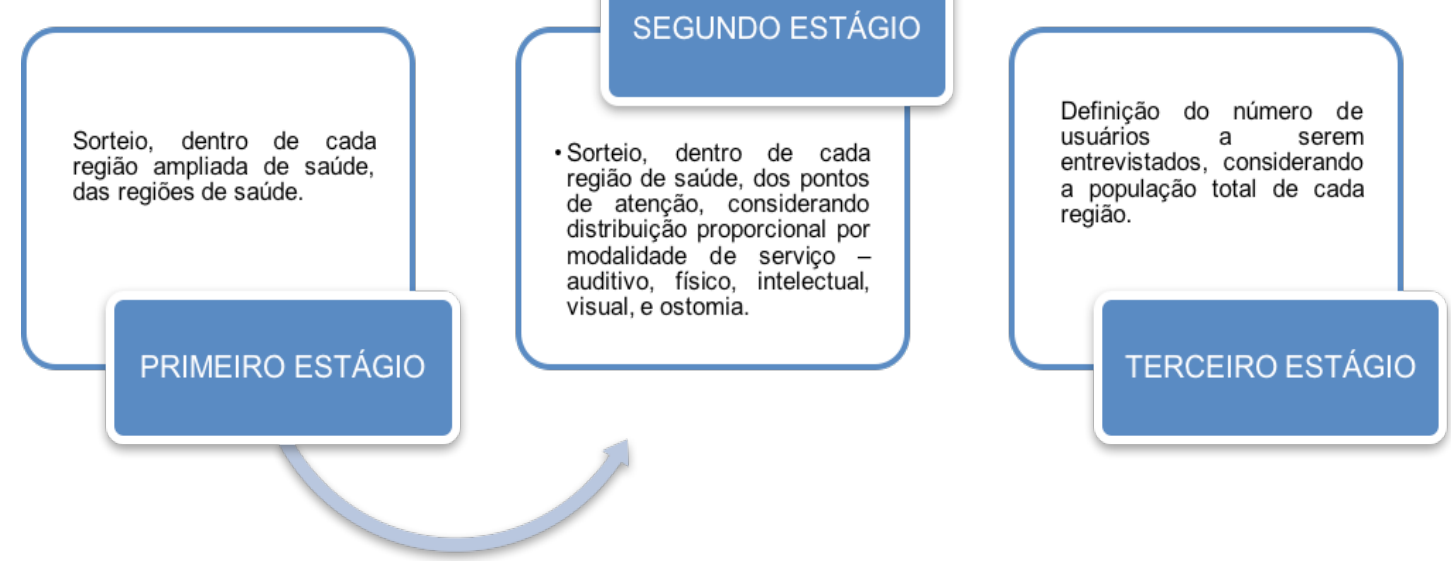

Figura 1. Estágios da amostragem aleatória

nível de significância $\alpha, \boldsymbol{\beta}$ é a margem de erro, $N$ o tamanho total da população com deficiência nas regiões de saúde e $p$ alguma proporção de interesse provinda do instrumento de pesquisa. Dessa forma, considerando-se a população total de 18.225 .658 e a prevalência de $14 \%$ de pessoas com deficiência, tem-se um $\mathrm{N}$ igual a 2.551.593, que representa a população total de pessoas com deficiência no estado de Minas Gerais.

$$
\mathrm{n}=\frac{\mathrm{N}}{\frac{(\mathrm{N}-1) \beta^{2}}{\mathrm{p}(1-\mathrm{p}) \mathrm{z}_{\alpha}{ }^{2}}+1}
$$

Para possibilitar o cálculo do tamanho da amostra para as diferentes variáveis com os níveis especificados de significância e margem de erro, foi utilizado um $p$ de $50 \%$, que representa a prevalência. Tal escolha é a suposição máxima possível e se justifica por não se conhecer todas as possibilidades das diferentes variáveis, uma vez que o tamanho da amostra obtido sobre esta suposição é máximo, suficiente para qualquer possível resultado que venha a ocorrer.

\section{Síntese do percurso metodológico}

Na figura 2 é apresentada a síntese do percurso metodológico utilizado para delineamento do projeto de pesquisa.

\section{Resultados preliminares}

Na primeira etapa foi realizado o mapeamento inicial da RCPD a partir da análise dos dados secundários fornecidos pela SES/MG. Foi considerada a presença dos pontos de atenção em todo território do estado, o tipo de serviço ofertado e sua relação com a densidade populacional. Para tanto foi realizado o georreferenciamento dos pontos de atenção e realizadas técnicas de geoprocessamento a fim de descrever a distribuição espacial do componente da atenção especializada, de acordo com a legislação nacional e estadual.

Além dos dados da SES-MG, foram utilizadas informações do Cadastro Nacional de Estabelecimentos de Saúde CNES/DATASUS, referentes ao componente especializado da RCPD do Estado de Minas Gerais. Foram identificados 209 serviços, distribuídos pelas modalidades: serviço de reabilitação da deficiência intelectual $(63,6 \%)$, reabilitação do paciente ostomizado (13,9\%), reabilitação física $(8,6 \%)$, reabilitação auditiva $(6,2 \%)$, reabilitação visual $(1,4 \%)$ e centros especializados em reabilitação (6,2\%). Nestes serviços, existem 3.271 profissionais cadastrados para compor as equipes mínimas de cada especialidade. No entanto, $17,7 \%$ dos serviços não apresentam a equipe mínima completa. Além disso, foi observado que há maior número de equipamentos voltados para a atenção 


\begin{tabular}{|c|c|c|}
\hline Etapa I: MAPEAMENTO DA REDE & 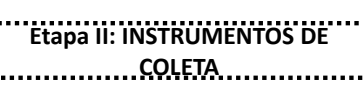 & $\begin{array}{l}\text { Etapa III: INSTRUMENTALIZAÇÃO E } \\
\text { ACOMPANHAMENTO DO PROJETO }\end{array}$ \\
\hline \multirow{2}{*}{$\begin{array}{l}\text { Dados gerais do mapeamento da } \\
\text { Rede: dados secundários } \\
\text { fornecidos pela SES } \\
\end{array}$} & \multirow{2}{*}{$\begin{array}{l}\text { Elaboração dos instrumentos de } \\
\text { coleta: usuários, } \\
\text { gestores/coordenadores; } \\
\text { profissionais; estrutura do serviço }\end{array}$} & $\begin{array}{l}\text { a) Estudo da temática pelo grupo e } \\
\text { definição do referencial teórico }\end{array}$ \\
\hline & & $\begin{array}{l}\text { b) Submissão e aprovação ao } \\
\text { Comitê de Ética }\end{array}$ \\
\hline \multirow{2}{*}{$\begin{array}{l}\text { Informações do estudo: dados } \\
\text { secundários do CNES }\end{array}$} & \multirow[b]{2}{*}{ Estudo piloto } & c) Seleção de bolsistas \\
\hline & & $\begin{array}{l}\text { d) Treinamento dos bolsistas para } \\
\text { o trabalho de campo }\end{array}$ \\
\hline
\end{tabular}

Figura 2. Síntese do percurso metodológico para delineamento do projeto de pesquisa

de pessoas com deficiência intelectual $(73,8 \%)$, além de maior concentração dos serviços na Região Ampliada de Saúde Sul (29,7\%).

Na próxima etapa, baseada na compilação de referenciais teóricos e de dados da RCPD, o grupo elaborou os instrumentos de coleta. Em consenso, ficou definido que os instrumentos deveriam ter como objetivo a avaliação da RCPD pelos diferentes atores e em distintas dimensões (assistência, gestão e estrutura). Deste modo, foram delineados instrumentos para realização de entrevistas com usuários, gestores/coordenadores dos serviços de saúde e profissionais, bem como análise da estrutura do serviço. (quadro 1)

Quadro 1. Instrumentos de coleta da pesquisa sobre RCPD/MG e seus objetivos

\begin{tabular}{|c|c|c|}
\hline Instrumento & Eixos e principais itens abordados & Tipo de elaboração \\
\hline Usuários & $\begin{array}{l}\text { Roteiro estruturado que tem como objetivo analisar a percepção } \\
\text { e satisfação dos usuários com os serviços e a Rede, de acordo } \\
\text { com os seguintes eixos temáticos: a) dados sociodemográficos e } \\
\text { econômicos; b) dados do cuidador/informante; c) caracterização } \\
\text { do acesso, percurso assistencial e utilização da Rede; d) satisfa- } \\
\text { ção com a Rede; e) avaliação objetiva da satisfação do usuário. }\end{array}$ & $\begin{array}{l}\text { Equipe de } \\
\text { pesquisadores }\end{array}$ \\
\hline Profissionais & $\begin{array}{l}\text { Roteiro estruturado com o objetivo de analisar a percepção do } \\
\text { profissional quanto a implementação da Rede de Cuidados à Pessoa } \\
\text { com Deficiência na região de abrangência do serviço em que atua, } \\
\text { bem como sua satisfação, por meio dos seguintes eixos temáticos } \\
\text { serão: a) dados sociodemográficos e econômicos; b) acesso; c) } \\
\text { rotina assistencial; d) satisfação com o serviço; e) recursos hu- } \\
\text { manos; e) participação em eventos científicos. }\end{array}$ & $\begin{array}{l}\text { Equipe de } \\
\text { pesquisadores }\end{array}$ \\
\hline $\begin{array}{l}\text { Gestor/Coordenador } \\
\text { do serviço }\end{array}$ & $\begin{array}{l}\text { Roteiro estruturado com objetivo de verificar a percepção do ges- } \\
\text { tor/coordenador do serviço quanto aos seguintes eixos temáticos: } \\
\text { a) dados sociodemográficos e econômicos; b) satisfação; c) pontos } \\
\text { positivos e negativos do serviço/Rede. }\end{array}$ & $\begin{array}{l}\text { Equipe de } \\
\text { pesquisadores }\end{array}$ \\
\hline Estrutura do serviço & $\begin{array}{l}\text { Roteiro de observação estruturada a ser preenchido pelos pesqui- } \\
\text { sadores quanto em visita aos serviços com o objetivo de descrever } \\
\text { e analisar a estrutura dos pontos de atenção, verificando se a } \\
\text { estrutura está de acordo com o preconizado na portaria (referencia } \\
\text { portaria). Seus eixos temáticos serão: a) identificação do serviço; } \\
\text { b) estrutura física; c) equipamentos; d) recursos humanos; e) } \\
\text { entorno do serviço. }\end{array}$ & $\begin{array}{l}\text { Equipe de } \\
\text { pesquisadores }\end{array}$ \\
\hline $\begin{array}{l}\text { Avaliação das redes } \\
\text { de atenção à saúde }\end{array}$ & $\begin{array}{l}\text { Instrumento proposto por } \text { Mendes }^{16} \text { (2011) e publicado pelo } \\
\text { Ministério da Saúde. }\end{array}$ & Literatura $^{16}$ \\
\hline
\end{tabular}


Para fins de calibração dos instrumentos elaborados e balizamento das escolhas metodológicas delineadas, foi realizado estudo piloto em um serviço da RCPD da região metropolitana de Belo Horizonte/MG, selecionado aleatoriamente. Dessa forma, foi possível identificar viabilidade, fragilidades e potenciais dificuldades de aplicação dos instrumentos, o que permitiu realizar ajustes e adequar os instrumentos para implementação da fase da pesquisa de campo. A aplicação dos instrumentos em um grupo semelhante ao alvo da pesquisa permite que o grupo de avaliadores perceba a maneira como pode abordar as pessoas a serem investigadas, amplia a afinidade com as questões, permite verificar a pertinência e adequação das mesmas quanto à forma de elaboração e vocabulário. Além de permitir que sejam estimados os tempos de aplicação dos instrumentos.

Após realização do cálculo amostral, foi definido o total de 385 usuários da RCPD, ao nível de significância de 5\%, divididos pelas 13 regiões ampliadas de saúde, conforme tabela 1 .

Tabela 1. Tamanho amostral por região ampliada de saúde e por modalidade de serviço

\begin{tabular}{|c|c|c|c|c|c|c|c|c|}
\hline \multirow{2}{*}{$\begin{array}{c}\text { Região Ampliada } \\
\text { de Saúde }\end{array}$} & \multicolumn{7}{|c|}{ N por modalidade do serviço da Região Ampliada de Saúde } & \multirow[b]{2}{*}{$\mathbf{N}$ total* } \\
\hline & SRV & SERDI & SASA & SRF & SASPO & CER & $\begin{array}{l}\text { SRF/ } \\
\text { SASPO }\end{array}$ & \\
\hline Centro & 5 & 59 & 21 & 10 & 11 & 6 & 5 & 117 \\
\hline Centro Sul & 1 & 9 & 0 & 2 & 2 & 0 & 2 & 16 \\
\hline Jequitinhonha & 0 & 3 & 0 & 0 & 0 & 3 & 0 & 6 \\
\hline Leste & 0 & 14 & 3 & 3 & 3 & 3 & 2 & 28 \\
\hline Leste do Sul & 0 & 8 & 2 & 0 & 1 & 2 & 2 & 15 \\
\hline Nordeste & 0 & 7 & 1 & 1 & 2 & 0 & 0 & 11 \\
\hline Noroeste & 0 & 8 & 0 & 0 & 0 & 3 & 2 & 13 \\
\hline Norte & 0 & 4 & 3 & 0 & 0 & 8 & 4 & 19 \\
\hline Oeste & 0 & 12 & 2 & 1 & 10 & 2 & 0 & 27 \\
\hline Sudeste & 0 & 22 & 1 & 2 & 6 & 1 & 0 & 32 \\
\hline Sul & 0 & 47 & 1 & 3 & 6 & 1 & 0 & 58 \\
\hline Triângulo do Norte & 0 & 16 & 1 & 2 & 6 & 2 & 0 & 27 \\
\hline Triângulo do Sul & 2 & 10 & 1 & 0 & 0 & 1 & 2 & 16 \\
\hline Total & 8 & 219 & 36 & 24 & 47 & 32 & 19 & 385 \\
\hline
\end{tabular}

Legenda: $*=\mathrm{n}$ total considerando margem de erro de 5\%; CER = Centro Especializado de Reabilitação; $\mathrm{N}=$ número de indivíduos; SASA = Serviço de Atenção à Saúde Auditiva; SASPO = Serviço de Atenção ao Paciente Ostomizado; SERDI = Serviço Especializado de Reabilitação de Deficiência Intelectual; SRF = Serviço de Reabilitação Física; SRV = Serviço de Reabilitação Visual.

\section{Instrumentalização e acompanhamento do projeto}

A etapa de instrumentalização e acompanhamento do projeto foi dividida em diversas fases, a saber:

a) apresentação da temática, nas primeiras reuniões, por duas integrantes com expertise na RCPD e atuantes na SES/MG, com foco em seus objetivos, complexidade e estruturação dentro do estado de Minas Gerais. Posteriormente, teve início o estudo de artigos referentes à temática, como forma de definir o referencial teórico a ser adotado na pesquisa;

b) Formatação da pesquisa para submissão e aprovação pelo Comitê de Ética em Pesquisa da
Universidade Federal de Minas Gerais (COEP/ UFMG).

c) Seleção de bolsistas, por meio de entrevista e análise de currículo. Foram selecionados: um profissional graduado em fonoaudiologia com mestrado em áreas afins (Bolsa de Gestão em Ciência e Tecnologia nível II) para ajudar no gerenciamento do projeto e atividades dos bolsistas; um profissional graduado em fonoaudiologia e com mestrado em áreas afins (Bolsa Apoio técnico nível I); três profissionais graduados em fonoaudiologia (Bolsa de Gestão em Ciência e Tecnologia Nível III) e dois graduandos de fonoaudiologia (Bolsa de Iniciação Científica).

d) Reuniões para apresentação da temática para 
os bolsistas e, posteriormente, realização de seminários para apresentação dos instrumentos elaborados, com o objetivo principal de determinar uma padronização em sua aplicação, evitando, assim, possíveis vieses na pesquisa. Foram realizadas reuniões periódicas para acom- panhamento permanente de todo o percurso da pesquisa.

Os objetivos previstos para a realização do projeto, bem como o estágio atual de realização estão descritos no quadro 2 .

Quadro 2. Objetivos previstos e estágio de realização

\begin{tabular}{|c|c|c|}
\hline Objetivos específicos & Atividade & Estágio \\
\hline $\begin{array}{l}\text { 1. Mapear a Rede de Cuidados à Pes- } \\
\text { soa com Deficiência em Minas Gerais, } \\
\text { identificando os pontos de atenção que } \\
\text { a compõem, sua área de abrangência } \\
\text { e os tipos de serviços ofertados por } \\
\text { cada um desses pontos. }\end{array}$ & $\begin{array}{l}\text { Análise dos dados secundários cedidos } \\
\text { pela Secretaria do Estado de Minas } \\
\text { Gerais pela equipe de pesquisa. }\end{array}$ & Concluído. \\
\hline $\begin{array}{l}\text { 2. Descrever a distribuição espacial } \\
\text { da Rede de Cuidados à Pessoa com } \\
\text { Deficiência no Estado de Minas Gerais. }\end{array}$ & $\begin{array}{l}\text { Análise dos dados secundários cedidos } \\
\text { pela Secretaria do Estado de Minas } \\
\text { Gerais pela equipe de pesquisa. }\end{array}$ & Concluído. \\
\hline $\begin{array}{l}\text { 3. Analisar a percepção dos profissio- } \\
\text { nais que compõem a Rede de Cuidados } \\
\text { à Pessoa com Deficiência acerca da } \\
\text { implantação da mesma na região de } \\
\text { abrangência do serviço em que atual } \\
\text { nos municípios selecionados. }\end{array}$ & $\begin{array}{l}\text { Aplicação de questionário semiestru- } \\
\text { turado elaborado pelos pesquisadores. }\end{array}$ & $\begin{array}{l}\text { Em fase de coleta de dados. Faltam a } \\
\text { compilação e análise dos dados. }\end{array}$ \\
\hline $\begin{array}{l}\text { 4. Analisar a estrutura física e os re- } \\
\text { cursos humanos da Rede de Cuidados } \\
\text { à Pessoa com Deficiência nos municí- } \\
\text { pios selecionados. }\end{array}$ & $\begin{array}{l}\text { Aplicação de questionário semiestru- } \\
\text { turado elaborado pelos pesquisadores. }\end{array}$ & $\begin{array}{l}\text { Em fase de coleta de dados. Faltam a } \\
\text { compilação e análise dos dados. }\end{array}$ \\
\hline $\begin{array}{l}\text { 5. Identificar os usuários que utilizam } \\
\text { a Rede de Cuidados à Pessoa com De- } \\
\text { ficiência nos municípios selecionados. }\end{array}$ & $\begin{array}{l}\text { Aplicação de questionário semiestru- } \\
\text { turado elaborado pelos pesquisadores. }\end{array}$ & $\begin{array}{l}\text { Em fase de coleta de dados. Faltam a } \\
\text { compilação e análise dos dados. }\end{array}$ \\
\hline $\begin{array}{l}\text { 6. Analisar a percepção de usuários } \\
\text { que utilizam a Rede de Cuidados à } \\
\text { Pessoa com Deficiência nos municípios } \\
\text { selecionados. }\end{array}$ & $\begin{array}{l}\text { Aplicação de questionário semiestru- } \\
\text { turado elaborado pelos pesquisadores. }\end{array}$ & $\begin{array}{l}\text { Em fase de coleta de dados. Faltam a } \\
\text { compilação e análise dos dados. }\end{array}$ \\
\hline $\begin{array}{l}\text { 7. Verificar a associação entre a per- } \\
\text { cepção dos profissionais, usuários e } \\
\text { estrutura dos serviços da Rede de } \\
\text { Cuidados à Pessoa com deficiência nos } \\
\text { municípios selecionados em relação } \\
\text { às características individuais, demo- } \\
\text { gráficas, socioeconômicas e ao Índice } \\
\text { Mineiro de Responsabilidade Social. }\end{array}$ & $\begin{array}{l}\text { Contratação de assessoria estatística } \\
\text { para análise dos dados. } \\
\text { Previamente, os dados coletados se- } \\
\text { rão digitados em um banco de dados } \\
\text { e conferidos para posterior análise. } \\
\text { Será realizada análise descritiva da } \\
\text { distribuição de frequência de todas } \\
\text { as variáveis categorias e análise de } \\
\text { medidas de tendência central e de dis- } \\
\text { persão das variáveis continuas. Para } \\
\text { análises de associação serão realiza- } \\
\text { dos os testes Qui-quadrado e Exato de } \\
\text { Fisher, considerando estatisticamente } \\
\text { significantes os que apresentarem } \\
\text { valor de p } \leq \leq 0,05 \text {. }\end{array}$ & $\begin{array}{l}\text { Em fase de coleta de dados. Faltam a } \\
\text { compilação e análise dos dados. }\end{array}$ \\
\hline $\begin{array}{l}\text { 8. Analisar a trajetória entre a residên- } \\
\text { cia e os serviços que compõem a Rede } \\
\text { de Cuidados à Pessoa com Deficiência } \\
\text { nos municípios selecionados. }\end{array}$ & $\begin{array}{l}\text { Dados obtidos por meio do questioná- } \\
\text { rio semiestruturado aplicado. A análise } \\
\text { será realizada por meio de contratação } \\
\text { de assessoria estatística. }\end{array}$ & $\begin{array}{l}\text { Em fase de coleta de dados. Faltam a } \\
\text { compilação e análise dos dados. }\end{array}$ \\
\hline
\end{tabular}


Vencidas estas etapas, as próximas contemplaram a pesquisa de campo, já em fase de realização, bem como produção de trabalhos científicos com os resultados obtidos.

\section{Discussão}

No campo da saúde coletiva, a descentralização e a regionalização das ações, diretrizes do SUS, são primordiais para o avanço da implementação e consolidação do sistema de saúde enquanto resposta às necessidades de saúde da população. Ao compartilhar as competências e responsabilidades entre os entes federados, permite-se que a organização de ações e serviços promovam a integralidade e equidade da assistência prestada, além de evitar o desperdício dos recursos empregados ${ }^{17-19}$. No entanto, tal processo é complexo e enfrenta diversas dificuldades, dentre elas, a de se obter o compromisso dos municípios pela real capacidade de prover o cuidado à população, de forma resolutiva e integrada em todos os níveis de atenção ${ }^{18}$. A compreensão da regionalização, não apenas como forma de racionalizar a oferta de serviços, pode ampliar esse debate ao se considerar, nesse processo, outros aspectos do território e suas dinâmicas econômicas, políticas, sociais e culturais. Nesse sentido, a regionalização pode ser vista como possibilidade de resposta local não circunscrita ao setor da saúde ${ }^{19}$. Portanto, para além do mapeamento inicial da Rede de Cuidados da Pessoa com Deficiência, que apresenta como sua organização o território e as regiões de saúde, é preciso avançar na compreensão das dinâmicas locais e das possíveis combinações do conjunto de serviços com outros arranjos externos ao setor da saúde.

Considerando-se a importância da avaliação em saúde para a formulação e implementação de políticas públicas e a metodologia desse projeto, que propõe a análise de três elementos (estrutura, processo e resultado $)^{20}$, os instrumentos da presente pesquisa foram elaborados pelos pesquisadores, com a finalidade de abordar e entender a organização local dos serviços, tendo como base a avaliação da estrutura, satisfação de usuários e profissionais e monitoramento da RCPD. Ressalta-se que para se estabelecer um processo avaliativo é essencial que sua construção apresente bases lógicas, coerentes e racionais, de forma a dar confiabilidade aos dados obtidos ${ }^{21}$. Cabe ainda destacar que a escolha pela abordagem avaliativa normativa se deve à abrangência da Rede, enquanto objeto desse estudo, os incipientes dados disponíveis na literatura e a necessidade de se produzir informações úteis ao gestor da RCPD. A apreciação normativa, embora não apresente elementos de uma avaliação participativa - denominada de quarta geração, é pertinente por apresentar finalidade estratégica e formativa $^{22,23}$. Nesse sentido, essa pesquisa será capaz de apoiar no planejamento e fornecer informações para melhorar a intervenção em curso.

Quanto aos instrumentos de coleta elaborados pelo grupo de pesquisa, a etapa do estudo piloto, anterior à fase da coleta, foi essencial para testar, avaliar, revisar e aprimorar os procedimentos propostos nos métodos. Essa etapa permitiu a identificação de fragilidades do instrumento e de sua aplicação, que puderam ser revistas antes da implementação concreta da pesquisa ${ }^{24}$, além de ter propiciado o dimensionamento do tempo gasto em cada aplicação. Esse processo de revisão dos instrumentos foi realizado por toda a equipe, permitindo sua construção e reconstrução a partir de múltiplos olhares e, ainda, minimizar dúvidas especificas da aplicação, a retomada e revisão de aspectos específicos da metodologia e das etapas do estudo ${ }^{25}$. Nesse contexto, foi possível garantir uma padronização mínima esperada na coleta de dados, já envolvendo os avaliadores no processo de capacitação para o uso do instrumento.

A elaboração do cálculo amostral é justificada, pois se pretende que o estudo seja representativo da população, visando, assim, que os resultados encontrados possibilitem fazer inferências sobre toda a rede. Os parâmetros utilizados no cálculo contribuíram para diminuir a probabilidade de que as diferenças encontradas entre os grupos aconteçam ao acaso ${ }^{26}$.

Na etapa de instrumentalização e acompanhamento do projeto, várias fases foram realizadas. Inicialmente, o foco foi a aproximação e discussão da temática escolhida, por meio de reuniões periódicas e estudo em grupo. Grande parte das pesquisas científicas realizadas, independente da área de conhecimento, tem como critérios de realização protocolos rígidos, definidos de acordo com o delineamento do estudo e o padrão do pesquisador ${ }^{27}$. No entanto, é imprescindível considerar a diversidade dos saberes do grupo, seja por meio de reuniões de discussão, grupos de estudo, rodas de conversa, oficinas ou outro meio. Assim, experiências e práticas individuais são apresentadas 
como forma de agregar conhecimento ao grupo/ pesquisa, visto que nenhum pesquisador é detentor único do saber ${ }^{27}$.

A definição do referencial teórico a ser adotado, também realizada nas fases iniciais, auxiliou na seleção de procedimentos e aspectos metodológicos a serem utilizados, uma vez que permitiu aproximação com a temática do estudo e definição de conceitos. Ressalta-se, no entanto, que embora algumas definições tenham sido feitas nas etapas iniciais, alterações foram realizadas no curso da execução do projeto, tendo em vista o avanço das discussões do grupo e os aspectos de viabilidade observados especialmente na fase de coleta de dados. Compreende-se, dessa forma, que é necessário algum grau de flexibilização frente ao planejamento em todas as etapas do projeto, uma vez que isso torna possível reformular e adequar o delineamento do seu estudo, aproximando-o de seu objetivo ${ }^{28}$. Tal prática permite inclusive que novos modelos/ percursos metodológicos sejam desenvolvidos e compartilhados com outros pares.

Após a aprovação do projeto pelo Comitê de Ética em Pesquisa, ocorreu a seleção de bolsistas para compor a equipe de pesquisa. Inicialmente foi selecionado o bolsista de Gestão em Ciência e Tecnologia (nível II), para fornecer o apoio técnico e auxiliar no gerenciamento das atividades do projeto e pesquisa de campo e, posteriormente, os demais bolsistas, com funções de apoio técnico e pesquisa de campo.

Quanto à seleção de bolsistas, foi observado que a inserção de novos integrantes ao grupo de pesquisa por meio de critérios claros, assim como a definição da função de cada um, favorece o estabelecimento de diretrizes bem definidas ao projeto, respeitando as diferenças, mas sem perder a característica de uma liderança segura e objetiva ${ }^{29}$. Outra dimensão importante é a de formação de novos pesquisadores, por meio da vivência de todas as etapas de um estudo, e da troca de experiências e saberes com orientadores, pesquisadores e demais integrantes. Ressalta-se, ainda, a relevância da inserção de bolsistas de iniciação científica em grupos de pesquisa, o que propicia ao graduando não apenas a vivência em práticas de pesquisa ${ }^{30}$, mas também a formação do seu senso crítico-reflexivo.

$\mathrm{Na}$ fase de instrumentalização dos bolsistas o principal objetivo foi a inserção dos novos integrantes na temática de estudo e também a padronização do processo de aplicação dos instrumentos de coleta. Foram realizadas discussões em grupo acerca da temática, seminários para explicação dos instrumentos elaborados e adotados e seminários para padronização da aplicação dos instrumentos ${ }^{25}$.

No momento da redação deste artigo, a presente pesquisa está em fase final de coleta de campo, mas já sistematizou resultados preliminares para apresentação em congressos e eventos científicos. Este momento também é muito importante para a equipe, que pode rever os processos estabelecidos e qualifica os bolsistas para a elaboração de resumos e artigos, apresentação de trabalhos, reflexão sobre a análise de dados.

\section{Conclusão}

A escolha pela descrição do percurso metodológico utilizado para o delineamento deste estudo permitiu aos pesquisadores sistematizar todo processo de elaboração e desenvolvimento de um projeto de pesquisa. Acredita-se que o estudo apresenta contribuições ao meio científico, pois relata as etapas traçadas por uma equipe para elaboração de seu projeto de pesquisa e os aspectos metodológicos utilizados. É de relevância o registro deste percurso para que novos estudos possam reproduzir este processo e, consequentemente, a comparação entre resultados obtidos em outros cenários. Espera-se que os resultados obtidos nas próximas etapas possam contribuir para a análise crítica da implementação da RCPD em Minas Gerais e seu impacto na qualidade de vida do usuário, visando o avanço da construção da integralidade da assistência e da adequação das políticas vigentes. Além disso, poderá fornecer subsídios para que medidas de melhoria possam ser adotadas na gestão da RCPD no âmbito estadual, de forma a promover a real integração entre gestores, profissionais de saúde e usuários.

\section{Referências}

1. OMS: Organização Mundial de Saúde. Classificação Internacional de Funcionalidade, Incapacidade e Saúde (CIF). Tradução e revisão Amélia Leitão. Lisboa: OMS, 2004, 238p. [acesso em 20 jul 2016]. Disponível em: http://www.inr.pt/ uploads/docs/cif/CIF_port_\%202004.pdf.

2. Junior AFC, Machiavelli JL. (Org.). Atenção e Cuidado da Saúde bucal da pessoa com deficiência: introdução ao estudo. Recife: Ed. Universitária; 2015. 65p. 
3. Castaneda L, Castro SS, Bahia L. Construtos de incapacidade presentes na pesquisa nacional por amostra de domicilio (PNAD): uma análise baseada na Classificação Internacional de Funcionalidade, Incapacidade e Saúde (CIF). Rev Bras Estud Popul. 2014; 31(2): 419-29.

4. Munguba MCS, Vieira ACVC, Porto CMV. Da invisibilidade à participação social: promoção da saúde em pessoas com deficiência. Rev Bras Promoc Saude. 2015; 28(4): 463-6.

5. OMS: Organização Mundial de Saúde. THE WORLD BANK. Relatório mundial sobre a deficiência. Trad. Lexicus Serviços Linguísticos. São Paulo: EDPcD; 2012. 334p.

6. IBGE: Instituto Brasileiro de Geografia e Estatística. Pesquisa Nacional de Saúde 2013 - Ciclos de vida - Brasil e grandes regiões. Rio de Janeiro: IBGE; 2015. 92p.

7. IBGE: Instituto Brasileiro de Geografia e Estatística. Censo Demográfico 2010. Disponível em: http://censo2010.ibge. gov.br/.

8. Brasil. Ministério da Saúde. Portaria n ${ }^{\circ} 793$, de 24 de abril de 2012. Institui a Rede de Cuidados à Pessoa com Deficiência no âmbito do Sistema Único de Saúde. Disponível em: http://bvsms.saude.gov.br/bvs/saudelegis/gm/2012/ prt0793_24_04_2012.html.

9. Gryschek ALFP, Nichiata LYI, Fracolli LA, Oliveira MAF, Pinho PH. Tecendo a rede de atenção à mulher em direção à construção da linha de cuidado da gestante e puérpera, no colegiado de gestão regional do Alto Capivari - São Paulo. Saude Soc. 2014; 23(2): 689-700.

10. Campos MF, Souza LAP, Mendes VLF. A rede de cuidados do Sistema Único de Saúde à saúde das pessoas com deficiência. Interface. 2015; 19(52): 207-10.

11. Holanda CMA, Queiroz ISA, Soares KA, Stolt LROG, Alves SB. O processo de construção da Rede da Pessoa com Deficiência no estado da Paraíba: um breve relato de experiência. Rev Bras Cienc Saude. 2014; 18: 33-8.

12. Minas Gerais. Secretaria de Estado de Saúde. Deliberação CIB-SUS/MG n ${ }^{\circ} 1.272$, de 24 de outubro de 2012. Institui a Rede de Cuidados à Pessoa com Deficiência SUS-MG e dá outras providências. Disponível em: http://www.saude.mg.gov. br/images/documentos/Deliberacao-1272redesdecuidadosPess oacomdeficiencia.pdf.

13. Minas Gerais. Secretaria de Estado de Saúde. Deliberação CIB-SUS/MG n 2003, de 09 de dezembro de 2014. Institui as atribuições e diretrizes de funcionamento das Juntas Reguladoras da Rede de Cuidados da Pessoa com Deficiência do SUS-MG (RCPD) e dá outras providências. Disponível em: http://www.saude.mg.gov.br/images/documentos/ Del2003DeliberacaoJRRCPD.pdf.

14. Malachias I, Leles FAG, Pinto MAS. Plano Diretor de Regionalização da Saúde de Minas Gerais. Belo Horizonte: Secretaria de Estado de Saúde de Minas Gerais; 2010. 264p.
15. Bolfarine H, Bussab W. Elementos de Amostragem. São Paulo: Edgar Blücher; 2005. 274p.

16. Mendes EV. As Redes de Atenção à Saúde. $2^{\mathrm{a}}$ ed. Brasília: Organização Pan Americana da Saúde; 2011. 549p.

17. Mendes A, Louvison MCP, Ianni AMZ, Leite MG, Feuerwerker LCM, Tanaka OY, et al. O processo de construção da gestão regional da saúde no estado de São Paulo: subsídios para análise. Saude Soc. 2015; 24(2): 423-27.

18. Mello ALSF, Andrade SR, Moysés SJ, Erdmann AL. Saúde bucal na rede de atenção e processo de regionalização. Cienc Saude Coletiva. 2014; 19(1): 205-14.

19. Ribeiro PT. Perspectiva territorial, regionalização e redes: uma abordagem à política de saúde da República Federativa do Brasil. Saude Soc. 2015; 24(2): 403-12.

20. Donabedia A. The quality of care-How can it be assessed? J Medic Care Org. 1988; 260(4): 1743-8.

21. Tanaka OY, Tamaki EM. O papel da avaliação para a tomada de decisão na gestão de serviços de saúde. Cienc Saude Coletiva. 2012; 17(4): 821-8.

22. Champagne $\mathrm{F}$ et al. A avaliação no campo da saúde: conceitos e métodos. In: Brousselle, A, Champagne F, Contandriopoulos AP, Hartz Z. Avaliação: conceitos e métodos. Rio de Janeiro: Editora Fiocruz; 2011. 292p.

23. Kantorski LP, Wetzel C, Olschowsky, Jardim VMR, Bielemann VLM, Schneider JF. Fourth-generation evaluation - methodological contributions for evaluating mental health services. Interface. 2009; 13(31): 343-55.

24. Bailer C, Tomitch LMB, D'Ely RCS. Planejamento como processo dinâmico: a importância do estudo piloto para uma pesquisa experimental em linguística aplicada. Rev Intercambio. 2011; XXIV: 129-46.

25. Goulart BNG, Martins-Reis VO, Chari BM. Inquérito domiciliar de distúrbios fonoaudiológicos autodeclarados: desenho e protocolo de pesquisa. ACR. 2015; 20(4): 336-48.

26. Patino, CM, Ferreira JC. Qual a importância do cálculo do tamanho amostral? J Bras Pneumol. 2016; 42(2): 162.

27. Cordeiro L, Soares CB, Campos CMS. Pesquisa ação na perspectiva da Saúde Coletiva: relato de experiência da formação de agentes comunitários da saúde para o enfrentamento do consumo prejudicial de drogas. Saude Transf Soc. 2013; 4(2): 105-16.

28. Lima TCS, Mioto RCT. Procedimentos metodológicos na construção do conhecimento científico: a pesquisa bibliográfica. Rev Katalysis. 2007; 10: 37-45.

29. Erdmann AL, Santos JLG, Klock P, Soder RM, Sasso GTMD, Erdmann RH. Políticas, gerência e inovação de grupos de pesquisa para a excelência em enfermagem. Aquichan. 2013; 13(1): 92-103.

30. Massi L, Queiroz SL. Estudos sobre iniciação científica no Brasil: uma revisão. Cad Pesq. 2010; 40(139): 173-97. 